\title{
Neutrino Oscillation Phase Shift from Quantum Gravity
}

\author{
Bipin Singh Koranga
}

Received: 12 April 2012 / Accepted: 11 July 2012 / Published online: 20 July 2012

(C) The Author(s) 2012. This article is published with open access at Springerlink.com

\begin{abstract}
The phase shift of neutrino oscillation could be discussed in the frame work of quantum gravity. Quantum gravity (Planck scale effects) leads to an effective $S U(2)_{L} \times U(1)$ invariant dimension-5 Lagrangian involving, neutrino and Higgs fields. On symmetry breaking, this operator gives rise to correction to the neutrino masses and mixing. We compute the neutrino oscillation phase due to Planck scale effects. The gravitational interaction $\left(M_{X}=M_{p l}\right)$ demands that the element of this perturbation matrix should be independent of flavor indices. In this paper, we study the quantum gravity effects on neutrino oscillation phases, namely modified dispersion relation for neutrino oscillation phases.
\end{abstract}

Keywords Neutrino oscillation phase shift · GUT scale

\section{Introduction}

Neutrinos are elementary neutral particles and are capable of passing through ordinary matter with minimum interaction. The existence of neutrino mass and mixings is experimentally well confirmed, therefore, the theoretical understanding of these quantities is one of the most important issues for Particle Physics. One of most sensitive probe of quantum gravity phenomena are neutrinos $[1,2]$. In recent year the subject of quantum gravity phenomenology has rapid growth complementary theoretical work. Theoretical extension of the standard model of Particle Physics, which also was expected to explain the origin and the shape of small neutrino mass matrix. Neutrino oscillations allow the transition among the three type of flavor eigenstates. The quantum mechanical phase of neutrinos, propagating in gravitational field has be discussed by many author [3,4]. The gravitational oscillation phase shift might have significant effect in supernova explosions, this result has been conformed in Ref. [5]. The outline of the article is as follows. In Sect. 2, we briefly discuss neutrino mass square difference and neutrino oscillation phase due to Planck scale effects. In Sect. 3, we discuss about numerical result. In Sect. 4, we present our conclusions.

B.S. Koranga $(\bowtie)$

Department of Physics, Kirori Mal College, University of Delhi, Delhi 110007, India

e-mail: bipiniitb@ rediffmail.com 


\section{Neutrino Mass Square Difference by Perturbation Approach}

The neutrino mass matrix is assumed to be generated by the Seesaw mechanism [7, 8]. The effective gravitational interaction of neutrino with Higgs field can be expressed as $S U(2)_{L} \times$ $U(1)$ invariant dimension-5 operator [6],

$$
L_{\text {grav }}=\frac{\lambda_{\alpha \beta}}{M_{p l}}\left(\psi_{A \alpha} \epsilon \psi_{C}\right) C_{a b}^{-1}\left(\psi_{B \beta} \epsilon_{B D} \psi_{D}\right)+\text { h.c. }
$$

Here and every where we use Greek indices $\alpha, \beta$ for the flavor states and Latin indices $i, j, k$ for the mass states. In the above equation $\psi_{\alpha}=\left(v_{\alpha}, l_{\alpha}\right)$ is the lepton doublet, $\phi=$ $\left(\phi^{+}, \phi^{o}\right)$ is the Higgs doublet, and $M_{p l}=1.2 \times 10^{19} \mathrm{GeV}$ is the Planck mass, $\lambda$ is a $3 \times 3$ matrix in a flavor space with each elements $O(1)$. The Lorentz indices $a, b=1,2,3,4$ are contracted with the charge conjugation matrix $C$ and the $S U(2)_{L}$ isospin indices $A, B$, $C, D=1,2$ are contracted with $\epsilon=i \sigma_{2}, \sigma_{m}(m=1,2,3)$ are the Pauli matrices. After spontaneous electroweak symmetry breaking the Lagrangian in Eq. (1) generated additional term of neutrino mass matrix

$$
L_{\text {mass }}=\frac{v^{2}}{M_{p l}} \lambda_{\alpha \beta} v_{\alpha} C^{-1} v_{\beta},
$$

where $v=174 \mathrm{GeV}$ is the $V E V$ of electroweak symmetric breaking. We assume that the gravitational interaction is "flavor blind" that is $\lambda_{\alpha \beta}$ is independent of $\alpha, \beta$ indices. Thus the Planck scale contribution to the neutrino mass matrix is

$$
\mu \lambda=\mu\left(\begin{array}{lll}
1 & 1 & 1 \\
1 & 1 & 1 \\
1 & 1 & 1
\end{array}\right),
$$

where the scale $\mu$ is

$$
\mu=\frac{v^{2}}{M_{p l}}=2.5 \times 10^{-6} \mathrm{eV} .
$$

We take Eq. (3) as perturbation to the main part of the neutrino mass matrix, that is generated by GUT dynamics. We treat $M$ as the unperturbed (0th order) mass matrix in the mass eigenbasis. Let $U$ be the mixing matrix at 0 th order. Then the corresponding 0 th order mass matrix) $M$ in flavor space given by

$$
\mathbf{M}=U^{*} \operatorname{diag}\left(M_{i}\right) U^{\dagger},
$$

where, $U_{\alpha i}$ is the usual mixing matrix and $M_{i}$, the neutrino masses is generated by Grand unified theory. Most of the parameter related to neutrino oscillation are known, the major expectation is given by the mixing elements $U_{e 3}$. We adopt the usual parametrization.

$$
\begin{aligned}
& \frac{\left|U_{e 2}\right|}{\left|U_{e 1}\right|}=\tan \theta_{12}, \\
& \frac{\left|U_{\mu 3}\right|}{\left|U_{\tau 3}\right|}=\tan \theta_{23}, \\
& \left|U_{e 3}\right|=\sin \theta_{13} .
\end{aligned}
$$


In term of the above mixing angles, the mixing matrix is

$$
U=\operatorname{diag}\left(e^{i f 1}, e^{i f 2}, e^{i f 3}\right) R\left(\theta_{23}\right) \Delta R\left(\theta_{13}\right) \Delta^{*} R\left(\theta_{12}\right) \operatorname{diag}\left(e^{i a 1}, e^{i a 2}, 1\right) .
$$

The matrix $\Delta=\operatorname{diag}\left(e^{\frac{1 \delta}{2}}, 1, e^{\frac{-i \delta}{2}}\right)$ contains the Dirac phase. This leads to CP violation in neutrino oscillation $a 1$ and $a 2$ are the so called Majoring phase, which effects the neutrino less double beta decay. $f 1, f 2$ and $f 3$ are usually absorbed as a part of the definition of the charge lepton field. Due to Planck scale effects on neutrino mixing the new mixing matrix can be written as [9]

$$
\begin{aligned}
& U^{\prime}=U(1+i \delta \theta), \\
& \left(\begin{array}{ccc}
U_{e 1} & U_{e 2} & U_{e 3} \\
U_{\mu 1} & U_{\mu 2} & U_{\mu 3} \\
U_{\tau 1} & U_{\tau 2} & U_{\tau 3}
\end{array}\right)+i\left(\begin{array}{ccc}
U_{e 2} \delta \theta_{12}^{*}+U_{e 3} \delta \theta_{23}^{*}, & U_{e 1} \delta \theta_{12}+U_{e 3} \delta \theta_{23}^{*}, & U_{e 1} \delta \theta_{13}+U_{e 3} \delta \theta_{23}^{*} \\
U_{\mu 2} \delta \theta_{12}^{*}+U_{\mu 3} \delta \theta_{23}^{*}, & U_{\mu 1} \delta \theta_{12}+U_{\mu 3} \delta \theta_{23}^{*}, & U_{\mu 1} \delta \theta_{13}+U_{\mu 3} \delta \theta_{23}^{*} \\
U_{\tau 2} \delta \theta_{12}^{*}+U_{\tau 3} \delta \theta_{23}^{*}, & U_{\tau 1} \delta \theta_{12}+U_{\tau 3} \delta \theta_{23}^{*}, & U_{\tau 1} \delta \theta_{13}+U_{\tau 3} \delta \theta_{23}^{*}
\end{array}\right) .
\end{aligned}
$$

Where $\delta \theta$ is a hermition matrix that is first order in $\mu$ [10]. The first order mass square difference $\Delta M_{i j}^{2}=M_{i}^{2}-M_{j}^{2}$, get modified [10] as

$$
\Delta_{i j}^{\prime}=\Delta_{i j}+2\left(M_{i} \operatorname{Re}\left(m_{i i}\right)-M_{j} \operatorname{Re}\left(m_{j j}\right)\right),
$$

where

$$
\begin{gathered}
m=\mu U^{t} \lambda U, \\
\mu=\frac{v^{2}}{M_{p l}}=2.5 \times 10^{-6} \mathrm{eV} .
\end{gathered}
$$

The change in the elements of the mixing matrix, which we parametrized by $\delta \theta[10]$, is given by

$$
\delta \theta_{i j}=\frac{i \operatorname{Re}\left(m_{j j}\right)\left(M_{i}+M_{j}\right)-\operatorname{Im}\left(m_{j j}\right)\left(M_{i}-M_{j}\right)}{\Delta M_{i j}^{\prime 2}} .
$$

The above equation determine only the off diagonal elements of matrix $\delta \theta_{i j}$. The diagonal element of $\delta \theta_{i j}$ can be set to zero by phase invariance. For degenerate neutrinos, $M_{3}-M_{1} \cong$ $M_{3}-M_{2} \gg M_{2}-M_{1}$, because $\Delta_{31} \cong \Delta_{32} \gg \Delta_{21}$.

\section{Neutrino Oscillation Phase due to Planck Scale Effects}

The typical scenario of just two neutrino states of definite mass $v_{1}$ and $v_{2}$, the relevant unitary mixing matrix is simply a $2 \times 2$ sub matrix of the general mixing $U$. If we allow for non zero mass, we can assume that the state $\left|v_{e}\right\rangle$ and $v_{\mu}$ mix to give new state $\left|v_{1}\right\rangle$ and $\left|v_{2}\right\rangle$ defined by

$$
\left(\begin{array}{l}
v_{\mu} \\
v_{e}
\end{array}\right)=\left(\begin{array}{cc}
\cos \theta & \sin \theta \\
-\sin \theta & \cos \theta
\end{array}\right)\left(\begin{array}{l}
v_{1} \\
v_{2}
\end{array}\right)
$$


$\left|v_{1}\right\rangle$ and $\left|v_{2}\right\rangle$ are the mass eigenstates or the propagation eigenstates, we can write the equation of motion is

$$
i \frac{d}{d t}\left(\begin{array}{l}
v_{1} \\
v_{2}
\end{array}\right)=H\left(\begin{array}{l}
v_{1} \\
v_{2}
\end{array}\right) .
$$

Since $\left|v_{1}\right\rangle$ and $\left|v_{2}\right\rangle$ are eigenstates of the Hamiltonian, the state $\left|v_{1}\right\rangle$ and $\left|v_{2}\right\rangle$ evolves as

$$
\begin{aligned}
& \left|v_{1}(t)\right\rangle=e^{-i E_{1} t}\left|\nu_{1}(t)\right\rangle, \\
& \left|v_{1}(t)\right\rangle=e^{-i E_{1} t}\left|\nu_{1}(t)\right\rangle .
\end{aligned}
$$

The probability of the in ital state $v_{e}$ oscillate in the state $v_{\mu}$ at a time $t$ is given by

$$
P\left(v_{e} \rightarrow v_{\mu}\right)=\sin ^{2} \theta \cos ^{2} \theta \cos \left(\left(E_{2}-E_{1}\right) t\right) .
$$

As neutrino is an extremely relativistic particle, we can make the assumption $E \gg m$

$$
E_{i}=\sqrt{p^{2}+m_{i}^{2}} \simeq p+\frac{m^{2}}{2 E} .
$$

The transition probability can finally be simplified

$$
P\left(v_{e} \rightarrow v_{\mu}\right)=\frac{1}{2} \sin ^{2} 2 \theta_{12}\left(1-\cos \left(\frac{\Delta_{21} L}{2 E}\right)\right),
$$

where $\Delta_{21}=m_{2}^{2}-m_{1}^{2}>0$ is the mass square differences. $E$ is the neutrino energy and $L$ is the baseline length.

The phase of neutrino oscillation in vacuum due to the interference of two massive neutrinos $v_{1}$ and $v_{2}$ with masses $m_{1}$ and $m_{2}$. The standard expression for the oscillation phase

$$
\phi_{G U T}=\left(\frac{1.27 \Delta_{21} L}{E}\right) \text {. }
$$

Approaches to quantum gravity that give rise to modified mass square difference from Eq. (11). The corresponding modified oscillation phase due to Planck scale effects, analogous to Eq. (17) is then given by

$$
\phi_{\text {Planck }}^{\prime}=\left(\frac{1.27 \Delta_{21}^{\prime} L}{E}\right) .
$$

We define the ratio $\phi$

$$
\begin{gathered}
\phi=\frac{\phi_{\text {Planck }}}{\phi_{G U T}}=\frac{\Delta_{21}^{\prime}}{\Delta_{21}}, \\
\phi_{\text {Planck }}=\phi_{G U T}\left(\frac{\Delta_{21}^{\prime}}{\Delta_{21}}\right) .
\end{gathered}
$$




\section{Numerical Results}

Note from Eq. (11) that the correction term depends on crucially the type of neutrino mass spectrum. For a hierarchical or inverse hierarchical spectrum the correction is negligible. Hence, we consider a degenerated neutrino Mass spectrum and take the common neutrino mass to be $2 \mathrm{eV}$, which is upper limit of tritium beta decay spectrum [11]. From definition of the matrix $m$ in Eq. (11), we find

$$
\begin{aligned}
& m_{11}=\mu e^{i 2 a_{1}}\left(U_{e 1} e^{i f 1}+U_{\mu 1} e^{i f 2}+U_{\tau 1} e^{i f 3}\right)^{2}, \\
& m_{22}=\mu e^{i 2 a_{2}}\left(U_{e 2} e^{i f 1}+U_{\mu 2} e^{i f 2}+U_{\tau 2} e^{i f 3}\right)^{2} .
\end{aligned}
$$

The contribution of the term in the Planck scale correction, $\epsilon=2\left(M_{i} \operatorname{Re}\left(m_{11}\right)-\right.$ $\left.M_{j} \operatorname{Re}\left(m_{22}\right)\right)$, can be additive or subtractive depending on the values of the phase $a_{1}, a_{2}$ and phase $f_{i}$. In our calculation, we used mixing angle as $\theta_{12}=34^{\circ}, \theta_{23}=45^{\circ}, \theta_{13}=10^{\circ}$, and $\delta=0^{\circ}$. We have taken $\Delta_{31}=0.002 \mathrm{eV}^{2}$ [12] and $\Delta_{21}=0.00008 \mathrm{eV}^{2}$ [13]. For simplicity, we have set the charge lepton phases $f_{1}=f_{2}=f_{3}=0$. Since we have set $\theta_{13}=0$, the Dirac phase $\delta$ drops out of the 0th order mixing matrix. In Table 1 we list the modified neutrino phase terms for some sample value of $a_{1}$ and $a_{2}$.

Table 1 The modified mass square difference and neutrino oscillation phase term for various value of phase. Input value are $\Delta_{31}=2.0 \times 10^{-3} \mathrm{eV}^{2}$, $\Delta_{21}=8.0 \times 10^{-8} \mathrm{eV}^{2}$, $\theta_{12}=34^{\circ}, \theta_{23}=45^{\circ}, \theta_{13}=0^{\circ}$

\begin{tabular}{rrll}
\hline$a_{1}$ & \multicolumn{1}{c}{$a_{2}$} & $\Delta_{21}^{\prime}$ & $\phi_{\text {Planck in term of } \phi_{G U T}}$ \\
\hline $0^{\circ}$ & $0^{\circ}$ & $7.6 \times 10^{-5} \mathrm{eV}^{2}$ & 0.75 \\
$0^{\circ}$ & $45^{\circ}$ & $7.3 \times 10^{-5} \mathrm{eV}^{2}$ & 0.91 \\
$0^{\circ}$ & $90^{\circ}$ & $6.9 \times 10^{-5} \mathrm{eV}^{2}$ & 0.86 \\
$0^{\circ}$ & $145^{\circ}$ & $7.3 \times 10^{-5} \mathrm{eV}^{2}$ & 0.91 \\
$0^{\circ}$ & $180^{\circ}$ & $7.6 \times 10^{-5} \mathrm{eV}^{2}$ & 0.95 \\
$45^{\circ}$ & $0^{\circ}$ & $8.3 \times 10^{-5} \mathrm{eV}^{2}$ & 1.03 \\
$45^{\circ}$ & $45^{\circ}$ & $8.3 \times 10^{-5} \mathrm{eV}^{2}$ & 1.03 \\
$45^{\circ}$ & $90^{\circ}$ & $7.6 \times 10^{-5} \mathrm{eV}^{2}$ & 0.95 \\
$45^{\circ}$ & $135^{\circ}$ & $7.9 \times 10^{-5} \mathrm{eV}^{2}$ & 0.98 \\
$45^{\circ}$ & $180^{\circ}$ & $8.3 \times 10^{-5} \mathrm{eV}^{2}$ & 1.03 \\
$90^{\circ}$ & $0^{\circ}$ & $9.0 \times 10^{-5} \mathrm{eV}^{2}$ & 1.12 \\
$90^{\circ}$ & $45^{\circ}$ & $8.6 \times 10^{-5} \mathrm{eV}^{2}$ & 1.07 \\
$90^{\circ}$ & $90^{\circ}$ & $8.3 \times 10^{-5} \mathrm{eV}^{2}$ & 1.03 \\
$90^{\circ}$ & $135^{\circ}$ & $8.6 \times 10^{-5} \mathrm{eV}^{2}$ & 1.07 \\
$90^{\circ}$ & $180^{\circ}$ & $9.0 \times 10^{-5} \mathrm{eV}^{2}$ & 1.12 \\
$135^{\circ}$ & $0^{\circ}$ & $8.3 \times 10^{-5} \mathrm{eV}^{2}$ & 1.03 \\
$135^{\circ}$ & $45^{\circ}$ & $7.9 \times 10^{-5} \mathrm{eV}^{2}$ & 0.98 \\
$135^{\circ}$ & $90^{\circ}$ & $7.6 \times 10^{-5} \mathrm{eV}^{2}$ & 0.95 \\
$135^{\circ}$ & $135^{\circ}$ & $7.9 \times 10^{-5} \mathrm{eV}^{2}$ & 0.98 \\
$135^{\circ}$ & $180^{\circ}$ & $8.3 \times 10^{-5} \mathrm{eV}^{2}$ & 1.03 \\
$180^{\circ}$ & $0^{\circ}$ & $7.6 \times 10^{-5} \mathrm{eV}^{2}$ & 0.95 \\
$180^{\circ}$ & $45^{\circ}$ & $7.3 \times 10^{-5} \mathrm{eV}^{2}$ & 0.91 \\
$180^{\circ}$ & $90^{\circ}$ & $6.9 \times 10^{-5} \mathrm{eV}^{2}$ & 0.86 \\
$180^{\circ}$ & $135^{\circ}$ & $7.3 \times 10^{-5} \mathrm{eV}^{2}$ & 0.91 \\
$180^{\circ}$ & $180^{\circ}$ & $7.6 \times 10^{-5} \mathrm{eV}^{2}$ & 0.95 \\
\hline & & &
\end{tabular}




\section{Conclusions}

It is expected that a higher scale generation the neutrino mass matrix, which will eventually produce the presently observed masses and mixings. In an attractive scenario,the mixing pattern generated by higher scale dynamics is predicted to be bi-maximal. We consider that the main part of neutrino masses and mixing from GUT scale operator. The gravitational interaction of lepton field with S.M. Higgs field give rise to a $S U(2)_{L} \times U(1)$ invariant dimension-5 effective Lagrangian give originally by Weinberg [8]. On electroweak symmetry breaking this operators leads to additional mass terms. We considered these to be perturbation of GUT scale mass terms. This model predict a value for the modified neutrino oscillation phase $\phi_{\text {planck }}^{\text {min }}=(1-0.25) \phi_{G U T}$ and $\phi_{\text {planck }}^{\text {max }}=(1+0.12) \phi_{G U T}$, which is correspondence to Planck scale $M_{p l} \approx 2.0 \times 10^{19} \mathrm{GeV}$. This occurs, of course for degenerate neutrino mass with a common mass of about $2 \mathrm{eV}$.

Open Access This article is distributed under the terms of the Creative Commons Attribution License which permits any use, distribution, and reproduction in any medium, provided the original author(s) and the source are credited.

\section{References}

1. Fogli, G.L., et al.: Phys. Rev. D 76, 033006 (2007)

2. Lisi, E., et al.: Phys. Rev. Lett. 85, 1166 (2000)

3. Ahluwalia, D.V., Burgard, C.: Gen. Relativ. Gravit. 28, 1161 (1996)

4. Ahluwalia, D.V., Burgard, C.: Phys. Rev. D, Part. Fields 57, 4724 (1998)

5. Grossman, Y., Lipkin, H.J.: Phys. Rev. D, Part. Fields 55, 2760 (1997)

6. Weinberg, S.: Phys. Rev. Lett. 43, 1566 (1979)

7. Mohapatra, R.N., et al.: Phys. Rev. Lett. 44, 912 (1980)

8. Coleman, S., Galshow, S.L.: Phys. Rev. D, Part. Fields 59, 116008 (1999)

9. Vissani, F., et al.: Phys. Lett. B 571, 209 (2003)

10. Koranga, B.S., Narayan, M., Uma Sankar, S.: Phys. Lett. B 665, 63 (2008)

11. Kraus, C., et al.: Eur. Phys. J. 33, 5805 (2004)

12. Hosaka, J., et al. (Super-Kamiokande Collaboration): Phys. Rev. D 74, 32002 (2006)

13. Hosaka, J., et al. (KamLAND Collaboration): Phys. Rev. Lett. 94, 08180 (2005) 